\title{
Entre fonction et profession : pour une nouvelle pensée de la communication des organisations
}

Patrick Lamarque

\section{(2) OpenEdition}

1 Journals

Édition électronique

URL : http://journals.openedition.org/communicationorganisation/1712

DOI : 10.4000/communicationorganisation. 1712

ISSN : $1775-3546$

Éditeur

Presses universitaires de Bordeaux

Édition imprimée

Date de publication : 1 mai 1994

ISSN : 1168-5549

Référence électronique

Patrick Lamarque, «Entre fonction et profession : pour une nouvelle pensée de la communication des organisations », Communication et organisation [En ligne], 5 | 1994, mis en ligne le 26 mars 2012

consulté le 19 avril 2019. URL : http://journals.openedition.org/communicationorganisation/1712

DOI : 10.4000/communicationorganisation. 1712

Ce document a été généré automatiquement le 19 avril 2019

(c) Presses universitaires de Bordeaux 


\title{
Entre fonction et profession : pour une nouvelle pensée de la communication des organisations
}

\author{
Patrick Lamarque
}

1 Partons d'un constat si souvent vérifié qu'il faut le regarder comme un principe : quand elle perdure, toute fonction se transforme en profession. A partir d'une méthodologie dont elle se dote, elle circonscrit son périmètre, forme ses spécialistes et se justifie comme une des finalités légitimes des institutions auxquelles elle participe.

2 Depuis le XIX ${ }^{e}$ siècle, cette même démarche a conduit l'université à s'organiser autour de spécialités, en droit (avec l'émergence du droit constitutionnel, des libertés publiques, du droit commercial, du droit du travail...), en médecine, puis enfin dans l'ensemble du champ académique. Dans l'entreprise, tel fut le cas de la fonction financière, entre les deux guerres, du marketing, autour du deuxième conflit mondial, puis des ressources humaines dans les années 1950-1960 et de la stratégie un peu plus tard. Quant à l'organisation du travail, c'est une question constamment renouvelée depuis Taylor, au début de ce siècle, voire depuis les saint-simonniens.

De ce point de vue, après être longtemps demeurée un «art du faire» confié le plus souvent à des gens plus connus pour leur entregent que pour leur souci méthodologique, la fonction de directeur de communication -apparue sous ce titre en France à partir de 1978 - se trouve aujourd'hui à la croisée des chemins.

4 C'est d'abord sa nécessite qui s'est imposée, à partir de la compétition totale - au sens où il y a des " guerres totales »- dans laquelle les entreprises sont plongées, à tous les niveaux et sur une scène devenue mondiale. Dans ce contexte, elles ont compris que leur notoriété, leur réputation, l'ensemble des éléments qui concourent au "capital image » constituait un enjeu majeur tant dans le quotidien de leurs activités (recrutement, relations avec les autorités publiques...) qu'à l'occasion d'événements importants ou de crises (fusion-acquisitions, privatisations, conflits sociaux, accidents technologiques, problèmes d'environnement...). À leur suite, mais très rapidement, les institutions 
publiques ont pris conscience de ce qu'elles ne pouvaient plus rester à l'écart d'un mouvement qui faisait des citoyens des clients justiciables de toutes les attentions dans la sphère marchande tandis qu'ils demeuraient des sujets - «administrés » ou " usagers » pour ce qui touchait au collectif. A cet égard, la décentralisation s'est révélée un accélérateur majeur de l'expansion des pratiques communicationnelles.

\section{Mode ou nécessité}

Mais nécessité ne fait pas légitimité. C'est ce dont témoigna un sondage publié à l'occasion du Top-Com 1992 par «l'Expression d'entreprise », duquel il ressortait que, si la fonction communication était considérée comme utile et généralement conduite par des responsables compétents, elle apparaissait surtout liée aux médias, donc relativement exotique à l'organisation. Cette situation comporte un risque de marginalisation auquel les intéressés, notamment ceux que réunit "Entreprises et Médias", sont désormais sensibles. Ils cherchent donc à y porter remède en fondant plus précisément leurs actions. A preuve, l'élaboration par cette association d'une Charte déontologique, en juin 1992 et, plus encore, le souci actuel de la plupart des directeurs de communication, corrélatif aux restrictions budgétaires dues à la crise économique, de justifier le plus strictement possible le moindre franc investi. Là, le défaut d'une méthodologie claire et communément admise se fait cruellement sentir.

Or, à l'heure actuelle encore, rien n'est totalement gagné. On a vu par exemple, une des grandes entreprises françaises de l'agro-alimentaire supprimer cette fonction au motif que son président représentait son meilleur porte-parole. Sans doute aussi a-t-on imaginé que, puisque "tout est communication», il fallait surtout laisser les "Monsieur Jourdain ", à chacun des niveaux de l'organisation, exprimer leur foi communicative. Cette idée est d'autant plus tentante qu'on souhaite ne rien entreprendre d'engageant. Car, en matière managériale, notre pays est un étonnant adepte de toutes les modes qui passent, qu'il aime adopter un instant pour ne les développer jamais.

Depuis la guerre plusieurs couches sédimentaires se sont ainsi empilées dans le tissu économique. Ce fut d'abord, dans les fourgons du plan Marshall, l'irruption de la théorie organisationnelle du staff and line, puis la «direction par les objectifs" et la « rationalisation des choix budgétaires ». Dans les années soixante-dix émergèrent la psychologie du travail et les relations humaines; vint ensuite le mouvement de réorganisation des tâches dont subsistent encore, ici ou là, quelques équipes semiautonomes. Au cours de la décennie suivante, fleurirent "flux tendus", "cercles de qualité » et " groupes d'expression », avant que projets puis «culture » d'entreprise ne prennent leurs place dans les esprits. Désormais, il n'est plus question que d'éthique, en même temps que de nouveaux outils de recrutement (astrologie, numérologie...), tandis que quelques décideurs imaginatifs peaufinent des moyens avancés de motivation (individualisation des salaires, stages « hors limites »...). Curieusement, hormis les ÉtatsUnis - où ces modes s'inventent - et la France - où elles se répliquent -, les autres pays industriels ne manifestent pas une pareille sensibilité à l'air du temps. Les japonais s'en tiennent à leurs cercles de qualité et aux flux tendus, les allemands à la co-gestion et les pays nordiques à leur « démocratie industrielle » fondée sur les analyses socio-techniques du Tavistock Institute de Londres.

Mais, par-delà les raisons économiques et techniques, la communication s'impose comme une nécessité surtout parce qu'elle est un phénomène consubstantiel à la socialité. Jürgen 
Habermas écrit d'elle qu'elle s'inscrit dans un " processus d'intercompréhension entre le monde et le monde vécu» (in, Morale et communication, conscience morale et activité communicationnelle, Paris, Cerf, 1986, 4, III). Aucun rapport à l'Autre, ni même aucune conscience de soi, n'échappe à cette relation dialogique qui fait de la communication un agir spécifique. Rien d'étonnant à cela d'ailleurs, si l'on veut bien considérer que dans « communication » le cum témoigne de la nécessité de l'avec. Si l'on pose comme principe qu'en société démocratique/développée on tend à substituer à la contrainte la conviction et l'engagement respectif de chacun dans le pacte social, les progrès des techniques d'échange sont une condition de son avancée. Or, si aujourd'hui encore un espace contient des marges importantes de progression, c'est bien celui de la vie au travail. Trop souvent, en effet, on a le sentiment que, l'homme est devenu un adulte respecté en tant que citoyen et consommateur, mais qu'il demeure un sujet doté d'une autonomie réduite dès qu'il franchit les portes de son bureau ou de son atelier. A l'heure où le travail raréfié va commencer à s'envisager dans une problématique de dignité plus que de survie, cette question - malgré le mutisme actuel des sans-emploi comme des actifs -est appelée à jouer un rôle central dans notre coopération sociale.

9 À cet égard, les femmes et les hommes de communication, quelle que soit leur place dans l'espace public, devraient remplir une fonction médiatrice essentielle. A condition bien sûr, d'envisager leur mission non comme une " prêtrise » qui les autoriserait à participer du «mystère " principal de l'époque, mais comme celle de techniciens de la fluidité sociale. Il leur faut pour cela développer une méthode rigoureuse et transparente qui prévienne les tentations comme les procès en sorcellerie, et concevoir une éthique exigeante pour maîtriser les risques que présente tout système nouveau doté de moyens puissants.

\section{S'arracher au marketing}

10 Au regard de sa méthode, la communication demeure encore une sous-catégorie du marketing. Le mot lui-même s'est généralisé dans les années soixante sous l'influence des publicitaires, désireux d'euphémiser leurs techniques encore empreintes de "réclame » en nous incitant à considérer leurs études de marché comme autant de "feedback » anticipés du public. Tout comme aujourd'hui quand la télévision fait intervenir en direct des panélistes interrogés par téléphone ou minitel, on voulait alors nous faire croire à «l'interactivité » de ces démarches quand il s'agissait de passer du marketing de l'offre à celui de la demande. Qui ne voit en effet que, sous le concept « d'image corporate », dont le directeur de communication est le gardien, gît l'idée marketing de la marque étendue à l'ensemble de l'entreprise considérée elle-même comme une manière de produit à médiatiser? C'est ce que démontre bien le manuel récemment publié par Patrick d'Humières, sous le titre de management de la communication (Paris, Eyrolles, 1993), dans lequel, même si l'auteur s'efforce de remettre "en cause le tout-marketing", il est contraint d'organiser sa pensée autour du concept de « marque entreprise » (!).

11 S'il faut une nouvelle pensée de la communication, c'est précisément parce qu'une entreprise ne se résume pas à ses seuls produits. Elle est aussi composée de femmes et d'hommes réunis autour d'une stratégie globale et c'est cette communauté, fédérée par une visée collective, qu'elle doit exprimer. Plutôt que d'analyser "l'image », concept qui explicite assez bien les comportements d'achat, mais qui traduit moins clairement les attitudes profondes sur lesquelles se fondent l'image elle-même, mais aussi l'adhésion à 
une collectivité, les croyances, voire tout engagement réel. L'enjeu paraît d'autant plus actuel que, depuis plus d'une année déjà, les études nous révèlent un profond déficit de confiance au sein même des entreprises.

12 C'est donc plus à l'identité des organisations que devrait s'attacher la communication, c'est-à-dire aux systèmes de valeurs qui les légitiment, elles et leurs membres, à occuper le territoire qu'ils occupent et à produire les biens ou services qu'ils offrent. Car c'est bien la légitimité à agir dans chacune de ses dimensions, qui constitue le fondement stratégique central pour une entreprise comme pour une institution. Légitime elle est crédible, tant sur ses marchés que vis-à-vis de ses partenaires et de ses collaborateurs; illégitime, elle est bridée dans ses mouvements, comme engluée et déjà battue.

Cette ré-orientation du management de la communication devrait la rapprocher des méthodes développées en stratégie d'entreprise et en ressources humaines, en facilitant une pédagogie de l'encadrement, notamment aux niveaux intermédiaires. Elle pourrait en acquérir une vue plus large, plus en interaction avec les forces et les acteurs en présence, afin de modifier progressivement ses pratiques. Ainsi aurait-elle intérêt à limiter tout ce que le marketing définit comme des "actions ", maîtrisées de bout en bout, totalement quantifiables, mais du coup limitées dans le temps et dans le champ de leur intervention (une opération de publicité sur le lieu de vente, par exemple), au profit de dispositifs animés de leur propre dynamique, implicant réellement leurs acteurs, et dont les effets se font sentir bien au-delà de l'époque et des lieux de leur survenance. On pourrait, par opposition, les nommer des actes de communication. Tendue vers le durable et le vital voire la mémoire collective -, cette discipline gagnerait alors en profondeur et en portée ce qu'elle perdrait en miroitements de surface et s'insérerait durablement comme une profession reconnue dans l'entreprise.

\section{L'éthique contre les dérives}

14 Ainsi équipé, le communicateur ne doit jamais oublier le citoyen et garder en mémoire les dangers que recèle sa technique comme toute autre, afin d'éviter les ravages qu'elle pourrait engendrer.

La communication actuelle va vite, instaurant une véritable tyrannie de l'urgence sur l'information, au point qu'un journaliste aujourd'hui est moins coupable d'avoir diffusé une fausse nouvelle que d'avoir commis un « ratage ». De là une vulnérabilité naturelle de nos systèmes de communication à la désinformation comme l'a montré le triste épisode de Timisoara. Non contente d'aller vite, elle se trouve de plus en plus soumise aux règles spectaculaires du spot publicitaire, désormais véritable paradigme du dire contemporain. Il en résulte partout, à l'école comme dans la vie professionnelle, une propension à la pensée « clipée » au détriment de la rationalité argumentative et cette dérive risque de nous conduire à perdre une part de notre emprise sur la vie.

Le communicateur ne doit pas oublier non plus qu'en toute hypothèse, sa technique demeure un artefact condamné à n'être pas le réel mais sa représentation. Toutefois, en intervenant sur le monde, l'artefact s'incorpore à lui et le transforme, contractant une responsabilité propre à l'occasion de ce processus de déréalisation du réel. C'est pourquoi toute communication doit développer une éthique de sa propre relativité, constatant qu'elle ne remplit pas sa fonction si elle n'a pas l'Autre en perspective à l'intérieur de son agir spécifique. C'est pourquoi exemples d'évidence - le même message ne se véhicule pas 
avec les mêmes mots ni par les mêmes canaux selon ses destinataires, ou encore la culture propre d'un pays ou d'une région impose certaines précautions à l'occasion des campagnes internationales.

17 Mais là où l'éthique devra se montrer particulièrement vigilante, c'est dans le contrôle des dérives technologiques à travers lesquelles la communication risque désormais de se trouver impliquée. Elles ont trait à la mondialisation instantanée des images et des données (le live intégral) et aux dangers de détournement de la vérité que comportent les technologies numériques et celles du virtuel. Bientôt, une information, une image, un son, ne seront plus les produits de supports matériels (le film, la bande vidéo...) mais de simples données binaires à l'intérieur d'ordinateurs. De là à les transformer en effaçant toute preuve, il n'y a qu'un pas que certains ont déjà franchi. Lors de la campagne électorale américaine qui opposa MM. Bush et Dukakis, par exemple, l'un des candidats demanda à un publicitaire américain, Bob Greenberg, de procéder à des transformations faciales de son rival, filmé à l'occasion de discours réels. Il s'agissait de réaliser des spots publicitaires, destinés à être diffusés sur des chânes locales, pour faire apparaître ce candidat comme un hystérique. Les films ont été réalisés, avec une "vérité » saisissante, et s'ils n'ont pas été diffusés, ce fut du fait d'une réticence de conscience de la part du publicitaire.

Or, rapidement, ces techniques seront disponibles pour des filmages en direct. Déjà, un procédé existe qui permettra sous peu de diffuser en direct, à partir de stades, des publicités adaptées à chaque diffuseur. Et ce que l'on pourra réaliser pour des réclames sera disponible pour altérer tous les éléments constituant ce que l'on appelle, pour peu de temps encore, le « réel ». On est ici très loin de la propagande des sergents recruteurs, mais en même temps très proches par les problématiques posées. Toutes ont trait au respect de la Vérité et à l'attention à l'Autre, vertus cardinales de la communication, demain plus encore qu'hier.

Véritable langue d'Ésope actuelle, la communication contemporaine peut être à la fois la pire et la meilleure des choses. La plus indispensable aussi. C'est pourquoi incombe à tous ceux qui se font ses agents une responsabilité particulière en foi de quoi, selon qu'elle aura été bien ou mal assumée, le monde qui vient nous sera vivable ou non.

\section{RÉSUMÉS}

La fonction communication n'a pas encore pris toute sa place dans les organisations. Encore Jeune, elle manque d'une méthodologie qui lui soit propre et reste trop à la remorque des concepts posés par le marketing. Dans la période présente, marquée par son recentrage sur les enjeux internes, elle gagnerait à entreprendre un effort qui permette de rapprocher son appareil théorique de celui élaboré par les ressources humaines, voire de celui de la stratégie.

Communication does not yet have an important function within organisations. Being a relatively new concept it lacks its own methodology and it is still too close to concepts laid down by marketing. At the present time it is marked by a certain recentering on stakes internal to an 
organisation whereas it would benefit if a certain effort were made to bring its theory closer to that which has been drawn up in the field of human resources; or even in the field of strategy.

\section{AUTEUR}

\section{PATRICK LAMARQUE}

Directeur de communication après avoir coordonné la communication gouvernementale au Service d'Information et de Diffusion du Premier ministre. Patrick Lamarque, ancien élève à l'ENA, consacre une part importante de son temps à l'enseignement et à la recherche. Méthodologue, il a développé des techniques qualitatives d'analyse de l'efficacité des campagnes institutionnelles télévisées. En 1993, il a publié, chez ESF Éditeur, Les désordres du Sens ; alerte sur les médias, les entreprises, la vie publique. 